\title{
Another Proof of Existence of Global Weak Solutions to 1D Pollutant Transport Model
}

\author{
Brahima ROAMBA
}

Correspondence: Laboratoire de Mathmatiques, d'Informatique et Applications (LaMIA), Universit Nazi BONI, 01 BP 1091 Bobo-Dioulasso, Burkina Faso; Institut Universitaire de Technologie (IUT), Universit Nazi BONI, 01 BP 1091 Bobo-Dioulasso, Burkina Faso.

Received: January 3, 2021 Accepted: February 15, 2021 Online Published: March 11, 2021

doi:10.5539/jmr.v13n2p31 URL: https://doi.org/10.5539/jmr.v13n2p31

\begin{abstract}
This paper is devoted to the study of pollutant transport model by water in dimension one. The model studied extend the results obtained in ( Roamba, Zabsonré \& Zongo, 2017). However, our model does not take into account cold pressure term and the quadratic friction term as in (Roamba, Zabsonré \& Zongo, 2017) which are considered regularizing terms to show the existence of global weak solutions of your model. Without these regularizing terms, we show the existence of global weak solutions in time with a periodic domain.
\end{abstract}

Keywords: shallow water equations, pollutant, viscosity, friction

\section{Introduction}

In this paper, we are interested to the study of a pollutant transport model in one dimension.

As a reminder, several studies are being done on pollutant transport models. The authors in ( Fernandez-Nieto, NarbonaReina \& Zabsonré, 2013) were the pawns in the formal derivation of a bilayer model coupling shallow water and Reynolds lubrication equations. From this derivation, the authors prove that our model verify a dissapative entropy inequality up to a second'order term. They compare the numerical results with the viscous bilayer shallow water model proposed in ( Narbona-Reina, Zabsonré, Fernandez-Nieto \& Bresch ).

The authors in (Roamba, Zabsonré \& Zongo), have proven the existence of global weak solution of a similar model derived in ( Fernandez-Nieto, Narbona-Reina \& Zabsonré, 2013). To achieve this, the authors have made a technical hypothesis on the height of water, namely the water layer is more important than the layer of the pollutant in the form

$$
h_{2} \leq h_{1},
$$

where $h_{1}, h_{2}$ represent respectively the pollutant and the water height.

And to overcome this condition, they have resorted to the addition of regularizing terms such as Van Der waals force in (Kitavsev, Laurençot \& Niethammer (2011)) and laminar friction term see (Marche (2005); Mellet \& Vasseur (2007); Roamba, Zabsonré \& Traoré (2016)) to show the existence of global weak solutions of the models considered. The model studied in (Roamba, Zabsonré \& Zango (2017)) are read:

$$
\begin{gathered}
\partial_{t} h_{1}+\partial_{x}\left(h_{1} u\right)=0, \\
\partial_{t}\left(h_{1} u\right)+\partial_{x}\left(h_{1} u^{2}\right)+\frac{1}{2} g \partial_{x} h_{1}^{2}-4 v \partial_{x}\left(h_{1} \partial_{x} u\right)+\frac{u_{1}}{\beta}-h_{1} \partial_{x}\left(\sigma \partial_{x}^{2} h_{1}-V\left(h_{1}\right)\right) \\
r g h_{1} \partial_{x} h_{2}+r g h_{2} \partial_{x}\left(h_{1}+h_{2}\right)+r_{1} h_{1}|u|^{2} u=0 . \\
\partial_{t} h_{2}+\partial_{x}\left(h_{2} u_{1}\right)-\varepsilon \partial_{x}^{2} h_{2}-\partial_{x}\left(\left(a h_{2}^{2}+\operatorname{ch}_{2}^{3}\right) \partial_{x} p_{2}\right)=0 .
\end{gathered}
$$

with

$$
\partial_{x} p_{2}=\rho_{2} g \partial_{x}\left(h_{1}+h_{2}\right) \quad \text { and } \quad V\left(h_{1}\right)=\frac{1}{h_{1}^{3}}-\frac{\alpha}{h_{1}^{4}} \quad(\alpha>0)
$$

where $(t, x) \in(0, T) \times] 0,1[$, 
and $h_{1}, h_{2}$ are respectively, the water and the pollutant heights, $u$ is the water velocity. The ratio of densities is denoted $r=\frac{\rho_{2}}{\rho_{1}}$ where $\rho_{1}$ and $\rho_{2}$ are respectively the densities of the water and the pollutant. $v$ is the kinematic viscosity; $g$ is the constant gravity.

The coefficients $\sigma, r_{1}$ and $\beta$ are respectively the coefficients of the interfaz tension, quadratic friction and positive slip length parameters. $a$ and $c$ are constants. $\alpha$ and $\varepsilon$ are positive constants.

The model studied in this paper does not take into account cold pressure term (Van Der Waals force) and the quadratic friction term as in (Roamba, Zabsonré \& Zango (2017)). It is written as follows:

$$
\begin{gathered}
\partial_{t} h_{1}+\partial_{x}\left(h_{1} u\right)=0, \\
\partial_{t}\left(h_{1} u\right)+\partial_{x}\left(h_{1} u^{2}\right)-4 v \partial_{x}\left(h_{1} \partial_{x} u\right)+r g \partial_{x}\left[h_{1}\left(h_{2}+\frac{1}{2 r} h_{1}\right)\right]+\frac{1}{2} r g \partial_{x} h_{2}^{2}-\sigma h_{1} \partial_{x}^{3} h_{1}+a u=0, \\
\partial_{t} h_{2}+\partial_{x}\left(h_{2} u\right)-\varepsilon \partial_{x}^{2} h_{2}=0 .
\end{gathered}
$$

where $(t, x) \in(0, T) \times] 0,1[$.

There are many results on the existence of solutions of the one-dimensional Navier Stokes equations. In (Bresch \& Desjardins (2003)), the authors proved the existence of global weak solutions for 2D viscous Shallow Water equations and convergence to quasi-geotrophic model. In the paper, the authors shown the control of the vaccum thanks to an entropy named BD-entropy, which was introduced firstly in (Bresch, Desjardins \& Lin (2003)). We note that the authors in (Bresch, Desjardins \& Gérard-Varet (2007); Toumbou, Roux \& Sene (2007)) have used this BD-entropy to get existence result of global weak solutions for Shallow-Water and viscous compressible Navier-Stokes equations. We have used this entropy in our work.

The authors in (Haspot (2018)) have proved a result of global strond solutions to the Navier-Stokes system with degenerate viscosity coefficient. This work has been developed in (Kang \& Vasseur (2020)).

We integrate their ideas to limit the water height.

We draw on the work done in (Constantin, Drivas, Nguyen \& Pasqualotto (2020); Haspot (2018); Kang \& Vasseur (2020)) to improve the results obtained in (Roamba, Zabsonré \& Zango (2017)), by showing global existence of weak solutions of one-dimensional pollutant transport model without resorting to cold pressure term and regularizing terms.

The rest of paper is organized as follows: In the Section 2, we give firstly the definition of global weak solutions, secondly we establish a classical energy inequality and the "mathematical BD entropy", which give some regularities on the unknowns. We also give an existence theorem of global weak solutions in the same section. The Section 3 contains the proof of the energies estimates and main existence result theorem.

We complete the system studied with the initial conditions

$$
\begin{gathered}
\left.h_{1}(0, x)=h_{1_{0}}(x), \quad h_{2}(0, x)=h_{2_{0}}(x), \quad\left(h_{1} u\right)(0, x)=\mathbf{m}_{0}(x) \quad \text { in }\right] 0,1[. \\
h_{1_{0}} \in L^{2}(0,1), \quad\left|h_{1_{0}}+h_{2_{0}}\right| \in L^{2}(0,1), \quad \partial_{x}\left(h_{1_{0}}\right) \in L^{2}(0,1), \\
\partial_{x} \mathbf{m}_{0} \in L^{1}(0,1), \quad \mathbf{m}_{0}=0 \quad \text { if } h_{1_{0}}=0 \\
\frac{\left|\mathbf{m}_{0}\right|^{2}}{h_{1_{0}}} \in L^{1}(0,1), \quad \log h_{1_{0}} \in L^{1}(0,1) .
\end{gathered}
$$

\section{Mains Results}

Definition 1. We say that $\left(h_{1}, h_{2}, u\right)$ is a weak solution of (6)-(8), with the initial conditions (9)-(10), verifying the entropy inequalities (14) and (16) if for all smooth test function $\phi=\phi(t, x)$ with $\phi(T,)=$.0 , we have:

$$
\begin{gathered}
h_{1_{0}} \phi(0, .)-\int_{0}^{T} \int_{0}^{1} h_{1} \partial_{t} \phi-\int_{0}^{T} \int_{0}^{1} h_{1} u \partial_{x} \phi=0 \\
-h_{2_{0}} \phi(0, .)-\int_{0}^{T} \int_{0}^{1} h_{2} \partial_{t} \phi-\int_{0}^{T} \int_{0}^{1} h_{2} u \partial_{x} \phi+\varepsilon \int_{0}^{T} \int_{0}^{1} \partial_{x} h_{2} \partial_{x} \phi=0, \\
h_{1_{0}} u_{0} \phi(0, .)-\int_{0}^{T} \int_{0}^{1} h_{1} u \partial_{t} \phi-\int_{0}^{T} \int_{0}^{1} h_{1} u^{2} \partial_{x} \phi+4 v \int_{0}^{T} \int_{0}^{1} h_{1} \partial_{x} u \partial_{x} \phi
\end{gathered}
$$




$$
\begin{aligned}
& +\sigma \int_{0}^{T} \int_{0}^{1}\left(\partial_{x}^{2} h_{1} \partial_{x} h_{1}\right) \phi+\sigma \int_{0}^{T} \int_{0}^{1} h_{1} \partial_{x}^{2} h_{1} \partial_{x} \phi+a \int_{0}^{T} \int_{0}^{1} u \phi \\
& -r g \int_{0}^{T} \int_{0}^{1} h_{1} h_{2} \partial_{x} \phi-\frac{1}{2} g \int_{0}^{T} \int_{0}^{1} h_{1}^{2} \partial_{x} \phi-\frac{1}{2} r g \int_{0}^{T} \int_{0}^{1} h_{2}^{2} \partial_{x} \phi
\end{aligned}
$$

Lemma 1. (Energy inequality) For classical solutions of the system (6) - (8), the following inequality holds:

$$
\begin{aligned}
\frac{d}{d t} \int_{0}^{1}\left[\frac{1}{2} h_{1}|u|^{2}+\frac{1}{2} g(1-r)\left|h_{1}\right|^{2}+\right. & \left.\frac{1}{2} r g\left|h_{1}+h_{2}\right|^{2}+\frac{1}{2} \sigma\left|\partial_{x} h_{1}\right|^{2}\right]+4 v \int_{0}^{1} h_{1}\left|\partial_{x} u\right|^{2} \\
& +a \int_{0}^{1}|u|^{2}+\frac{1}{2} g r \varepsilon \int_{0}^{1}\left|\partial_{x} h_{2}\right|^{2} \leq \frac{1}{2} r g \varepsilon \int_{0}^{1}\left|\partial_{x} h_{1}\right|^{2} .
\end{aligned}
$$

Remark 1. Notice that the term in the right of (14) can be controlled using Gronwall's lemma.

Corollary 1. Let $\left(h_{1}, h_{2}, u\right)$ be a solution of model $(6)-(8)$. Then, thanks to Lemma 1 we have:

$\sqrt{h_{1}} u \quad$ is bounded in $\quad L^{\infty}\left(0, T ; L^{2}(0,1)\right), \quad \sqrt{h_{1}} \partial_{x} u \quad$ is bounded in $\quad L^{2}\left(0, T ; L^{2}(0,1)\right)$,

$u \quad$ is bounded in $\quad L^{2}\left(0, T ; L^{2}(0,1)\right), \quad\left(h_{1}+h_{2}\right) \quad$ is bounded in $\quad L^{\infty}\left(0, T ; L^{2}(0,1)\right)$,

$\partial_{x} h_{1} \quad$ is bounded in $\quad L^{\infty}\left(0, T ; L^{2}(0,1), \quad \partial_{x} h_{2} \quad\right.$ is bounded in $\quad L^{2}\left(0, T ; L^{2}(0,1)\right)$,

$h_{1} \quad$ is bounded in $\quad L^{\infty}\left(0, T ; L^{2}(0,1)\right), \quad h_{2} \quad$ is bounded in $\quad L^{\infty}\left(0, T ; L^{2}(0,1)\right)$.

Corollary 2. (see(Haspot (2018)))

There exists a constant $C>0$ such as

$$
h_{1} \geq C
$$

We will need in the following some additional regularity on $h_{1}$ and this will be achieved through an additional BD entropy inequality presented in the next lemma

Lemma 2. (BD-entropy) For smooth solutions $\left(h_{1}, h_{2}, u\right)$ of model (6) - (8) satisfying the classical energy equality of the Lemma 1, we have the following mathematical BD entropy inequality:

$$
\begin{gathered}
\frac{d}{d t} \int_{0}^{1}\left[\frac{1}{2} h\left|u+4 v \partial_{x} \log \left(h_{1}\right)\right|^{2}-4 v a \log \left(h_{1}\right)+\frac{1}{2} g(1-r)\left|h_{1}\right|^{2}+\frac{1}{2} r g\left|h_{1}+h_{2}\right|^{2}+\frac{1}{2} \sigma\left|\partial_{x} h_{1}\right|^{2}\right] \\
+a \int_{0}^{1}|u|^{2}+4 v \int_{0}^{1}\left(g+g r \frac{h_{2}}{h_{1}}\right)\left|\partial_{x} h_{1}\right|^{2}+r g \int_{0}^{1}\left(\varepsilon+4 v \frac{h_{2}}{h_{1}}\right) \partial_{x} h \partial_{x} h_{2} \\
+4 \sigma v \int_{0}^{1}\left|\partial_{x}^{2} h_{1}\right|^{2}+g r \varepsilon \int_{0}^{1}\left|\partial_{x} h_{2}\right|^{2} \leq \frac{1}{2} r g \varepsilon \int_{0}^{1}\left|\partial_{x} h_{1}\right|^{2} .
\end{gathered}
$$

While waiting to give the proof of the Lemma 2, we show how to control the term $\int_{0}^{T} \int_{0}^{1}\left(\varepsilon+4 v \frac{h_{2}}{h_{1}}\right) \partial_{x} h \partial_{x} h_{2}$, then all the others are good sign.

Indeed,

We have, $\frac{h_{2}}{h_{1}} \partial_{x} h_{1} \partial_{x} h_{2}=\left(\partial_{x} \log h_{1}\right) h_{2} \partial_{x} h_{2}$, we can write:

$$
\int_{0}^{T} \int_{0}^{1}\left|\frac{h_{2}}{h_{1}} \partial_{x} h_{1} \partial_{x} h_{2}\right| \leq \frac{1}{2} \int_{0}^{T} \int_{0}^{1}\left|\partial_{x} \log \left(h_{1}\right)\right|^{2}+\frac{1}{2} \int_{0}^{T} \int_{0}^{1}\left|h_{2} \partial_{x} h_{2}\right|^{2} .
$$

We will now look at the two terms to the right of the above inequality separately. For the first one, we have:

$$
\begin{aligned}
\int_{0}^{T} \int_{0}^{1}\left|\partial_{x} \log \left(h_{1}\right)\right|^{2}=\int_{0}^{T} & \int_{0}^{1} \frac{\left|\partial_{x} h_{1}\right|^{2}}{h_{1}^{2}} \\
& \leq \frac{1}{C^{2}} \int_{0}^{T} \int_{0}^{1}\left|\partial_{x} h_{1}\right|^{2} \quad(\text { see equation }(15)) .
\end{aligned}
$$


So

$$
\partial_{x}\left(\log h_{1}\right) \text { is in } L^{2}\left(0, T ; L^{2}(0,1)\right) \text {. }
$$

For the second one, since $h_{2} \in L^{\infty}\left(0, T ; H^{1}(0,1)\right)$ and $\partial_{x} h_{2} \in L^{2}\left(0, T ; L^{2}(0,1)\right)$ then, $h_{2} \partial_{x} h_{2} \in L^{2}\left(0, T ; L^{2}(0,1)\right)$ (By Sobolev embedding (see (Marche (2005)) for instance)) which completes the proof.

Corollary 3. Let $\left(h_{1}, h_{2}, u\right)$ be a solution of model (6) - (8).

Then, thanks to Lemma 2, we have:

$$
\sqrt{h_{1}}, \quad \partial_{x} \sqrt{h_{1}} \quad \text { are bounded in } \quad L^{\infty}\left(0, T ; L^{2}(0,1)\right) \quad \text { and } \quad \partial_{x}^{2} h_{1} \quad \text { is bounded in } \quad L^{2}\left(0, T ; L^{2}(0,1)\right)
$$

Remark 2. In the Corollary 1, the estimate

$$
\sqrt{h_{1}} u \quad \text { is bounded in } \quad L^{\infty}\left(0, T ; L^{2}(0,1)\right),
$$

implies,

$$
h_{1} u \quad \text { is bounded in } \quad L^{\infty}\left(0, T ; L^{2}(0,1)\right) \text {, }
$$

this lead us

$$
\begin{aligned}
& \partial_{t} h_{1} \quad \text { is bounded in } \quad L^{\infty}\left(0, T ; W^{-1,2}(0,1)\right), \\
& h_{1} \text { and } \sqrt{h_{1}} \text { are bounded in } L^{\infty}\left(0, T ; L^{\infty}(0,1)\right) .
\end{aligned}
$$

Corollary 4. The Remark 2 and the Corollary 2, allows us to state the following result, there exists constants $0<C$ and $R$, such as:

$$
0<C \leq h_{1} \leq R .
$$

Remark 3. We have the following additional regularities:

1. $h_{2}$ is bounded in $L^{2}\left(0, T ; L^{\infty}(0,1)\right) \cap L^{\infty}\left(0, T ; L^{2}(0,1)\right)$,

2. $u$ is bounded in $L^{2}\left(0, T ; L^{\infty}(0,1)\right) \cap L^{\infty}\left(0, T ; L^{2}(0,1)\right)$.

Theorem 1. There exists a global weak solutions to the system (6)-(8) with initial data (9)-(10), and satisfying energy enequalities (14) and (16).

\section{Proof of the Energies Inequalities and Theorem 1}

In this section we give proof of some results.

\subsection{Proof of Lemma 1}

First, we multiply the momentum equation by $u$ and we integrate from 0 to 1 .

$$
\begin{gathered}
\int_{0}^{1} \frac{1}{2} \partial_{t}\left(h_{1} u^{2}\right)+\frac{1}{2} \int_{0}^{1} g u \partial_{x} h_{1}^{2}-4 \int_{0}^{1} u \partial_{x}\left(v h_{1} \partial_{x} u\right)+r g \int_{0}^{1} h_{1} u \partial_{x} h_{2} \\
+r g \int_{0}^{1} h_{2} u \partial_{x}\left(h_{1}+h_{2}\right)+a \int_{0}^{1}|u|^{2}=0 .
\end{gathered}
$$

We use the mass conservation equation (6) for simplification. Then, we obtain:

$$
\begin{aligned}
-4 \int_{0}^{1} u \partial_{x}\left(v h_{1} \partial_{x} u\right) & =4 v \int_{0}^{1} h_{1}\left(\partial_{x} u\right)^{2} \\
--\sigma \int_{0}^{1} h_{1} u \partial_{x}^{3} h_{1}= & \sigma \int_{0}^{1} \partial_{x}\left(h_{1} u\right) \partial_{x}^{2} h_{1} \\
= & -\sigma \int_{0}^{1} \partial_{t} h_{1} \partial_{x}^{2} h_{1} \\
= & \sigma \int_{0}^{1} \partial_{x t} h_{1} \partial_{x} h_{1} \\
= & \frac{1}{2} \sigma \int_{0}^{1} \partial_{t}\left|\partial_{x} h_{1}\right|^{2} .
\end{aligned}
$$


- $r g \int_{0}^{1} h_{1} u \partial_{x} h_{2}=-r g \int_{0}^{1} h_{2} \partial_{x}\left(h_{1} u\right)=r g \int_{0}^{1} h_{2} \partial_{t} h_{1}$.

- $\frac{1}{2} g \int_{0}^{1} u \partial_{x} h_{1}^{2}=\frac{1}{2} g \int_{0}^{1} \partial_{t}\left|h_{1}\right|^{2}$.

- $r g \int_{0}^{1} h_{2} \partial_{x}\left(h_{1}+h_{2}\right) u=-r g \int_{0}^{1}\left(h_{1}+h_{2}\right) \partial_{x}\left(h_{2} u\right)$.

The equation for the thin film flow give us : $\partial_{x}\left(h_{2} u\right)=-\partial_{t} h_{2}+\varepsilon \partial_{x}^{2} h_{2}$ and we have:

- $r g \int_{0}^{1} h_{2} \partial_{x}\left(h_{1}+h_{2}\right) u=r g \varepsilon \int_{0}^{1} \partial_{x} h_{1} \partial_{x} h_{2}+r g \varepsilon \int_{0}^{1}\left|\partial_{x} h_{2}\right|^{2}+\frac{1}{2} r g \frac{d}{d t} \int_{0}^{1}\left|h_{2}\right|^{2}+r g \int_{0}^{1} h_{1} \partial_{t} h_{2}$

Substituting all these terms in (17), we get (14) by integrating under 0 to $T$.

\subsection{Proof of Lemma 2}

Let us multiply (7) by $4 v \partial_{x} \log (h)$, integrate with respect to $x$ and use an integration by parts. Thanks to the equation (6) and the initial conditions $(9)-(10)$, we have:

$$
\begin{gathered}
4 v \int_{0}^{1}\left(\partial_{t} u+u \partial_{x} u\right) \partial_{x} h_{1}+4 v g \int_{0}^{1}\left|\partial_{x} h_{1}\right|^{2}+16 v^{2} \int_{0}^{1} h_{1} \partial_{x} u \partial_{x}\left(\frac{\partial_{x} h_{1}}{h_{1}}\right)+4 a v \int_{0}^{1} \frac{u \partial_{x} h_{1}}{h_{1}}+4 \sigma v \int_{0}^{1}\left|\partial_{x}^{2} h_{1}\right|^{2}+ \\
+4 v_{1} r g \int_{0}^{1} \partial_{x} h_{2} \partial_{x} h_{1}+4 v_{1} r g \int_{0}^{1} \frac{h_{2}}{h_{1}}\left|\partial_{x} h\right|^{2}+4 v r g \int_{0}^{1} \frac{h_{2}}{h_{1}} \partial_{x} h_{2} \partial_{x} h_{1}=0 .
\end{gathered}
$$

On the one hand, a further integration by parts of the first term in (18), equation (6), and the energy inequality (14) give:

$$
\begin{aligned}
& 4 v \int_{0}^{1}\left(\partial_{t} u+u \partial_{x} u\right) \partial_{x} h_{1} \\
& =4 v\left(\frac{d}{d t} \int_{0}^{1} u \partial_{x} h_{1}-\int_{0}^{1} u \partial_{x t} h_{1}+\int_{0}^{1} u \partial_{x} u \partial_{x} h_{1}\right) \\
& =4 v\left(\frac{d}{d t} \int_{0}^{1} u \partial_{x} h_{1}-\int_{0}^{1} \partial_{x} u \partial_{x}\left(h_{1} u\right)+\int_{0}^{1} u \partial_{x} u \partial_{x} h_{1}\right) \\
& =4 v\left(\frac{d}{d t} \int_{0}^{1} u \partial_{x} h_{1}-\int_{0}^{1} h_{1}\left(\partial_{x} u\right)^{2}\right) \\
& =\frac{d}{d t} \int_{0}^{1}\left[4 v u \partial_{x} h_{1}+\frac{1}{2} h_{1}|u|^{2}+\frac{1}{2} g(1-r)\left|h_{1}\right|^{2}+\frac{1}{2} r g\left|h_{1}+h_{2}\right|^{2}+\frac{1}{2} \sigma\left|\partial_{x} h_{1}\right|^{2}\right] \\
& +a \int_{0}^{1}|u|^{2}+r g \varepsilon \int_{0}^{1} \partial_{x} h_{1} \partial_{x} h_{2}+r g \varepsilon \int_{0}^{1}\left|\partial_{x} h_{2}\right|^{2} .
\end{aligned}
$$

We can write the third and the fourth term in (18) as follow:

$$
\begin{gathered}
-16 v^{2} \int_{0}^{1} \partial_{x}\left(\frac{\partial_{x} h_{1}}{h_{1}}\right) \partial_{x} u h_{1}=\frac{1}{2} \frac{d}{d t} \int_{0}^{1} h_{1}\left|4 v \log \left(h_{1}\right)\right|^{2} \\
\bullet 4 a v \int_{0}^{1} \frac{u \partial_{x} h_{1}}{h_{1}}=4 a v \int_{0}^{1} \frac{\partial_{x}\left(u h_{1}\right)}{h_{1}}-4 a v \int_{0}^{1} \partial_{x} u \\
=-4 a v \frac{d}{d t} \int_{0}^{1} \log \left(h_{1}\right) \quad(\text { See ([?]) }
\end{gathered}
$$

Substituting finally the last three identities into (18), we obtain (16).

\subsection{Proof of Theorem 1}

This section is devoted to the prove of Theorem 1 . Let $\left(h_{1}^{k}, h_{2}^{k}, u^{k}\right)$ be a sequence of weak solutions with initial data

$$
h_{1 \mid t=0}^{k}=h_{1_{0}}^{k}, \quad h_{2 \mid t=0}^{k}=h_{2_{0}}^{k}, \quad\left(h_{1}^{k} u^{k}\right)_{\mid t=0}=m_{0}^{k}
$$

such that

$$
h_{1_{0}}^{k} \longrightarrow h_{1_{0}} \text { in } L^{1}(\Omega), \quad h_{2_{0}}^{k} \longrightarrow h_{2_{0}} \text { in } L^{1}(\Omega), \quad m_{0}^{k} \longrightarrow m_{0} \text { in }\left(L^{1}(\Omega)\right)^{2}
$$


and satisfies

$$
4 v \int_{0}^{1} \partial_{x} \log \left(h_{1_{0}}^{k}\right)+\int_{0}^{1}\left[h_{1_{0}}^{k}\left|u_{0}^{k}\right|^{2}+\frac{1}{2} g(1-r)\left|h_{1_{0}}^{k}\right|^{2}+\frac{1}{2} r g\left|h_{1_{0}}^{k}+h_{2_{0}}^{k}\right|^{2}+\frac{1}{2} \sigma\left|\partial_{x} h_{1_{0}}^{k}\right|^{2}\right] \leq C .
$$

Such approximate solutions can be built by a regularization of capillary effect.

3.3.1 Strong Convergence of $\sqrt{h_{1}^{k}}, \quad h_{1}^{k}$ and $h_{2}^{k}$

We first give the spaces in which $\sqrt{h_{1}^{k}}$ is bounded.

By integrating the mass equation, we obtain : $\sqrt{h_{1}^{k}}$ in $L^{\infty}\left(0, T ; L^{2}(0,1)\right)$.

As Remark 3 gives us $\partial_{x} \sqrt{h_{1}^{k}}$ in $L^{\infty}\left(0, T ; L^{2}(0,1)\right)$,

so

$$
\sqrt{h_{1}^{k}} \text { is bounded in } L^{\infty}\left(0, T ; L^{\infty}(0,1)\right) \text {. }
$$

Moreover, still using the mass equation, we obtain the following equality:

$$
\begin{aligned}
\partial_{t} \sqrt{h_{1}^{k}}= & \frac{1}{2} \sqrt{h_{1}^{k}} \partial_{x} u^{k}-\partial_{x}\left(\sqrt{h_{1}^{k}} u^{k}\right), \\
& =\frac{1}{2} \sqrt{h_{1}^{k}} \partial_{x} u^{k}-u^{k} \partial_{x} \sqrt{h_{1}^{k}}-\sqrt{h_{1}^{k}} \partial_{x} u^{k}
\end{aligned}
$$

which gives that $\partial_{t} \sqrt{h_{1}^{k}}$ is bounded in $L^{2}\left(0, T ; L^{2}(0,1)\right)$.

Applying Aubin-Simon lemma (Lions (1969); Simon (1987)), we can extract a subsequence, still denoted $\left(h_{1}^{k}\right)_{1 \leq k}$, such that

$$
\sqrt{h_{1}^{k}} \text { strongly converges to } \sqrt{h_{1}} \text { in } L^{2}\left(0, T ; L^{2}(0,1)\right) .
$$

According to the Corollary 4, we show that

$$
\left|h_{1}^{k}-h_{1}\right| \leq \sqrt{c_{2}}\left|\sqrt{h_{1}^{k}}-\sqrt{h_{1}}\right| \Rightarrow\left|h_{1}^{k}-h_{1}\right|^{2} \leq c_{2}\left|\sqrt{h_{1}^{k}}-\sqrt{h_{1}}\right|^{2} .
$$

This ensure

$$
h_{1}^{k} \quad \text { strongly converges to } h_{1} \text { in } L^{2}\left(0, T ; L^{2}(0,1)\right) \text {. }
$$

We have $h_{2}^{k} \in L^{2}\left(0, T ; L^{\infty}(0,1)\right)$. Moreover, we have $\partial_{t} h_{2}{ }^{k}=-\partial_{x}\left(h_{2}^{h} u^{k}\right)+\varepsilon \partial_{x}^{2} h_{2}$.

We have $h_{2}^{k} \in L^{\infty}(0,1)$ and $u^{k} \in L^{2}(0,1)$, so $h_{1}^{k} u^{k} \in L^{2}(0,1)$, according to the Sobolev embeddings, we show that the first term is in $W^{-1,2}(0,1)$. By analogy we prove that the last term is in the same space and we also get $\partial_{t} h_{2}^{k}$ in this space. Thanks to the Aubin-Simon lemma, we find:

$$
h_{2}^{k} \quad \text { strongly Converges to } h_{2} \text { in } L^{2}\left(0, T ; W^{-1,2}(0,1)\right) .
$$

3.3.2 Strong Convergence of $h^{k} u_{1}^{k}$ and $u^{k}$

We have $h_{1}^{k}$ that's bounded in $L^{\infty}\left(0, T ; L^{\infty}(0,1)\right)$ and $u^{k}$ that's bounded in $L^{2}\left(0, T ; L^{\infty}(0,1)\right)$, What gives us $h_{1}^{k} u^{k}$ is bounded in $L^{2}\left(0, T ; L^{\infty}(0,1)\right)$.

Let's look now $\partial_{x}\left(h_{1}^{k} u^{k}\right)$. We have:

$$
\partial_{x}\left(h_{1}^{k} u^{k}\right)=h_{1}^{k} \partial_{x} u^{k}+u^{k} \partial_{x} h_{1}^{k},
$$

based on the estimates obtained on $h_{1}^{k}$ in Remark 3, we get:

$$
\left(h_{1}^{k} u_{1}^{k}\right)_{k} \text { bounded in } L^{2}\left(0, T ; W^{1,2}(0,1)\right) .
$$

Moreover, the momentum equation (7) enables us to write the time derivation of the water discharge:

$$
\partial_{t}\left(h_{1}^{k} u^{k}\right)=-\partial_{x}\left(h_{1}^{k} u_{1}^{k^{2}}\right)-\frac{1}{2} g \partial_{x} h_{1}^{k^{2}}+4 v_{1} \partial_{x}\left(h_{1}^{k} \partial_{x} u\right)-a u^{k}+\sigma h_{1}^{k} \partial_{x}^{3} h_{1}^{k}-r g h_{1}^{k} \partial_{x} h_{2}^{k}-r g h_{2}^{k} \partial_{x}\left(h_{1}^{k}+h_{2}^{k}\right)
$$


we then study each term:

- $\partial_{x}\left(h_{1}^{k}\left(u^{k}\right)^{2}\right)=\partial_{x}\left(h_{1}^{k} u^{k}\left(u^{k}\right)\right)$ which is in $L^{2}\left(0, T ; W^{-1,2}(0,1)\right)$.

- as $h_{1}^{k}$ is in $L^{\infty}\left(0, T ; L^{\infty}(0,1)\right)$, and $\partial_{x} h_{1}^{k}$ is in $L^{2}\left(0, T ; L^{2}(0,1)\right)$ and we can write the following relation :

- $\partial_{x}\left[\left(h_{1}^{k}\right)^{2}\right]$ is bounded in $L^{2}\left(0, T ; L^{2}(0,1)\right)$.

- $\partial_{x}\left(h_{1}^{k} \partial_{x} u^{k}\right)$ is bounded in $L^{2}\left(0, T ; W^{-1,2}(0,1)\right)$.

- The last four terms are bounded in $L^{\infty}\left(0, T ; W^{-1,2}(0,1)\right)$.

Then, applying Aubin-Simon lemma, we obtain,

$\left(h_{1}^{k} u^{k}\right)_{k}$ stongly Converges to $h_{1} u$ in $C^{0}\left(0, T ; W^{-1,2}(0,1)\right)$.

3.3.3 Strong Convergence of $u^{k}, h_{1}^{k} \partial_{x} u^{k}$ and $\sqrt{h^{k}} u^{k}$

Thanks to Corollary 4 and Remark 3, we have $u^{k}$ and $\partial_{x} u^{k}$ are bounded in $L^{2}\left(0, T, L^{2}(0,1)\right)$. In order to obtain new estimates on $u^{k}$, we are going to control the right hand side of the following equation:

$\partial_{t} u^{k}=-u^{k} \partial_{x} u^{k}+4 v \partial_{x} \log h_{1}^{k} \partial_{x} u^{k}+4 v \partial_{x}^{2} u^{k}-g \partial_{x} h_{1}^{k}-r g \partial_{x} h_{2}^{k}-r g h_{2}^{k} \partial_{x} \log h_{1}^{k}-r g \frac{h_{2}}{h_{1}} \partial_{x} h_{2}^{k}+\sigma \partial_{x}^{3} h_{1}^{k}$.

Thanks to the estimates obtained on $h_{1}^{k}, h_{2}^{k}$ and $u^{k}$, all the terms to the right of equality except the last term are in $L^{2}\left(0, T, L^{2}(0,1)\right)$.

On the other hand, $\partial_{x}^{2} h_{1}^{k}$ is bounded in $L^{2}\left(0, T, L^{2}(0,1)\right)$, this lead us to $\partial_{x}^{3} h_{1}^{k}$ is bounded in $L^{2}\left(0, T, W^{-1,2}(0,1)\right)$ Aubin Simon's lemma leads us to the following result:

$$
\left(u^{k}\right)_{k} \text { stongly converges to } u \text { in } L^{2}\left(0, T ; W^{-1,2}(0,1)\right) \text {. }
$$

However, the function $\left(h_{1}^{k}, \partial_{x} u^{k}\right) \longmapsto h_{1}^{k} \partial_{x} u^{k}$ is continous in $L^{\infty}\left(0, T ; L^{\infty} 1(0,1)\right) \times L^{2}\left(0, T ; L^{2}(0,1)\right)$ to $L^{2}\left(0, T ; L^{2}(0,1)\right)$. So,

$$
h_{1}^{k} \partial_{x} u^{k} \quad \text { weakly converges to } \quad h_{1} \partial_{x} u \quad \text { in } L^{2}\left(0, T ; L^{2}(0,1)\right) .
$$

Thanks to the Corollary 4, we say that's exists constants $0<\alpha$ and $\beta<+\infty$ such as $\alpha \leq h_{1}^{k} \leq \beta$.

For all constant $k>\beta$, we have the following norm:

$$
\int_{0}^{T} \int_{0}^{1}\left|\sqrt{h_{1}^{k}} u^{k}-\sqrt{h_{1}} u\right|^{2} \leq \kappa \int_{0}^{T}\left|u^{k}-u\right|^{2} \longrightarrow 0
$$

So,

$$
\sqrt{h_{1}^{k}} u^{k} \quad \text { strongly converges to } \quad \sqrt{h_{1}} u \quad \text { in } \quad L^{2}\left(0, T ;\left(L^{2}(\Omega)\right)^{2}\right) \text {. }
$$

3.3.4 Strong Convergence of $\partial_{x} h_{1}^{k}, \quad h_{2}^{k} \partial_{x} h_{1}^{k}, \quad \partial_{x}^{2} h_{1}^{k}, \quad h_{1}^{k} \partial_{x}^{2} h_{1}$ and $\partial_{x} h_{1}^{k} \partial_{x}^{2} h_{1}^{k}$

- We have $\partial_{x} h_{1}^{k}$ bounded in $L^{2}\left(0, T ; H^{1}(0,1)\right)$ and $\partial_{t} \partial_{x} h_{1}^{k}$ is bounded in $L^{\infty}\left(0, T ; H^{-2}(0,1)\right)$ since $\partial_{t} h_{1}^{k}$ is bounded in $L^{\infty}\left(0, T ; H^{-1}(0,1)\right)$. Thanks to compact injection of $H^{1}(0,1)$ in $L^{2}(0,1)$ in one dimension, we have:

$$
\partial_{x} h_{1}^{k} \quad \text { strongly converges to } \quad \partial_{x} h \quad \text { in } L^{2}\left(0, T ; L^{2}(0,1)\right)
$$

- The bound of $\partial_{x}^{2} h_{1}^{k}$ in $L^{2}\left(0, T ; L^{2}(0,1)\right)$ and $\partial_{x} h_{2}^{k}$ in $L^{2}\left(0, T ; L^{2}(0,1)\right)$ gives us:

$$
\begin{array}{lll}
\partial_{x}^{2} h_{1}^{k} \quad \text { weakly converges to } & \partial_{x}^{2} h_{1} \quad \text { in } L^{2}\left(0, T ; L^{2}(0,1)\right), \\
\partial_{x} h_{2}^{k} \quad \text { weakly converges to } & \partial_{x} h_{2} \text { in } L^{2}\left(0, T ; L^{2}(0,1)\right) .
\end{array}
$$

- Thanks to the strong convergence of $h_{1}^{k}, h_{2}^{k}, \partial_{x} h_{1}^{k}$ and the weak convergence of $\partial_{x}^{2} h_{1}^{k}$, we have:

$$
h_{2}^{k} \partial_{x} h_{1}^{k} \quad \text { strongly converges to } \quad h_{2} \partial_{x} h_{1} \quad \text { in } L^{1}\left(0, T ; L^{1}(0,1)\right),
$$




$$
\begin{array}{rlll}
h_{1}^{k} \partial_{x}^{2} h_{1}^{k} & \text { strongly converges to } & h_{1} \partial_{x}^{2} h \quad \text { in } L^{1}\left(0, T ; L^{1}(0,1)\right), \\
\partial_{x} h_{1}^{k} \partial_{x}^{2} h_{1}^{k} & \text { strongly converges to } & \partial_{x} h_{1} \partial_{x}^{2} h_{1} \quad \text { in } L^{1}\left(0, T ; L^{1}(0,1)\right), \\
h_{1}^{k} \partial_{x} h_{2}^{k} & \text { strongly converges to } & h_{1} \partial_{x} h_{2} \quad \text { in } L^{1}\left(0, T ; L^{1}(0,1)\right), \\
h_{2}^{k} \partial_{x} h_{2}^{k} & \text { strongly converges to } & h_{2} \partial_{x} h_{2} \quad \text { in } L^{1}\left(0, T ; L^{1}(0,1)\right), \\
\left(h_{1}^{k}\right)^{2} & \text { strongly converges to } & h_{1}{ }^{2} \quad \text { in } L^{1}\left(0, T ; L^{1}(0,1)\right) .
\end{array}
$$

3.3.5 Convergences of $h_{2}^{k} u^{k}$ and $\partial_{x}^{2} h^{k}$

We know that $\partial_{x} h_{2}^{k}$ is bounded in $L^{2}\left(0, T ; L^{2}(0,1)\right)$ this implies $\partial_{x}^{2} h_{2}^{k}$ is in $L^{1}\left(0, T ; W^{-1,2}(0,1)\right)$. So,

$$
\partial_{x}^{2} h_{2}^{k} \quad \text { weakly converges to } \quad \partial_{x}^{2} h_{2} \in L^{1}\left(0, T ; W^{-1,2}(0,1)\right)
$$

To end we have $u^{k}$ weakly converges to $u$ in $L^{2}\left(0, T ; L^{2}(0,1)\right)$ and the strong convergence of $h_{2}^{k}$ to $h_{2}$, gives us:

$$
h_{2}^{k} u^{k} \quad \text { weakly converges to } \quad h_{2} u \quad \text { in } L^{1}\left(0, T ; L^{1}(0,1)\right) .
$$

\section{Conclusion}

In this paper, we show the existence of global weak solutions of a 1D pollutant transport model. We note that, for the model studied in this paper we did not take into account regularizing terms (cold pressure and quadratic friction terms) as in ( Roamba, Zabsonré \& Zongo, 2017) and we considered a better transport equation than that used in ( Roamba, Zabsonré \& Zongo, 2017).

\section{Acknowledgments}

The author sincerely thanks Professor Jean De Dieu ZABSONRE for the discussions related to the model and the theoretical aspect.

\section{References}

Bresch, D., \& Desjardins, B. (2003). Existence of global weak solution for 2D viscous shallow water equations and convergence to the quasi-geostrophic model. Comm. Math. Phys., 238(1-3), 211-223. https://doi.org/10.1007/s00220-003-0859-8

Bresch, D., Desjardins, B., \& Gérard-Varet, D. (2007). On compressible Navier-Stokes equations with density dependent viscosities in bounded domains. J. Math. Pures Appl., 87(9) , 227-235. https://doi.org/10.1016/j.matpur.2006.10.010

Bresch, D., Desjardins, B., \& Lin, C. K. (2003). On some compressible fluid models :Korteweg, lubrication and shallow water systems, Communications in partial differential equations, 28(3,4), 843-868. https://doi.org/10.1081/PDE-120020499

Constantin, P., Drivas T. D., Nguyen, T. D., \& Pasqualotto, F. (2020). Compressible fluide and active potentials. Annales de l'institut Henri Poincaré, 37(1), 145-180. https://doi.org/10.1016/j.anihpc.2019.04.001

Fernandez-Nieto, E. D., Narbona-Reina, G., \& Zabsonré, J. D. (2013). Formal derivation of a bilayer model coupling shallow water and Reynolds lubrication equations: evolution of a thin pollutant layer over water. European Journal of Applied Mathematics, 24(6), 803-833. https://doi.org/10.1017/S095679251300020X

Haspot, B. (2018). Existence of glabal strong solution for the compressible Navier-Stokes equation with degenerate viscosity coefficients 1D. Math. Nachr, 291(14-15), 2183-2203.

Kang, M. J., \& Vasseur, A. F. (2020). Global smooyh solutions for 1D bartropic Navier-Stokes equation with a large class of degenerate vicosities. Journal of nonlinear science. https://doi.org/10.1007/s00332-020-09622-z

Kitavtsev, G., Laurençot, P., \& Niethammer, B. (2011). Weak solutions to lubrication equations in the presence of strong slippage. Methods and Applications of Analysis, 18, 183-202. https://doi.org/10.4310/MAA.2011.v18.n2.a4

Marche, F. (2007). Theoretical and Numerical Study of Shallow Water Models. Applications to Nearshore Hydrodynamics. PhD Thesis, University of Bordeaux .

Mellet, A., \& Vasseur, A. (2007). On the barotropic compressible Navier-Stokes equations. Comm. Partial Differential Equations, 32(3), 431-452. http://dx.doi.org/10.1080/03605300600857079 
Narbona-Reina, G., Zabsonré, J. D., Fernandez-Nieto, E. D., \& Bresch, D. (2009). Derivation of a bilayr shallow water model with viscosity. Numerical validation. Comput. Model. Eng. Sci., 4(41), 27-71. http://dx.doi:10.397/cmes.2009.043.027

Toumbou, B., Le Roux, D., \& Sene, A. (2007). An existence theorem for a 2-D coupled sedimentation shallow-water model. C. R. Math. Acad. Sci. Paris, 344, 443-446. https://doi.org/10.1016/j.jde.2007.12.010

Lions, J. L. (1969). Quelques Methodes de Resolution des Problemes aux Limites Non Line aires, Dunod.

Roamba, B., Zabsonré, J. D. D., \& Traoré, S. (2016). Formal derivation and existence of global weak solutions of a two-dimensional bilayer model coupling shallow water and Reynolds lubrication equations. Asymptotic Analysis, 99, 207-239. https://doi.org/10.3233/ASY-16138

Roamba, B., Zabsonré, J. D. D., \& Zongo, Y. (2017). Weak solutions to pollutant transport model in a dimensional case. Annals of the University of Craiova, Mathematics and Computer Science Series, 44(1), 137-148. Retrieved from http://inf.ucv.ro/ ami/index.php/ami

Roamba, B., Zabsonré, J. D. D., \& Zongo, Y. (2017). On the existence of global weak solutions to 1D pollutant transport model. Journal of Mathematics Research, 9(4). https://doi.org/ 10.5539/jmr.v9n4p124

Simon, J. (1987). Compact set in the space $L^{p}(0 ; t ; B)$. Ann. Mat. Pura appl., 146(4), 65-96.

Zabsonré, J. D. D., Lucas, C., \& Fernandez-Nieto, E. (2009). An energetically consistent viscous sedimentation model. Math. Models Methods Appl. Sci., 19(3), 477-499. https://dx.doi.org/10.1142/S0218202509003504

\section{Copyrights}

Copyright for this article is retained by the author(s), with first publication rights granted to the journal.

This is an open-access article distributed under the terms and conditions of the Creative Commons Attribution license (http://creativecommons.org/licenses/by/4.0/). 